\title{
An explicit description of the second cohomology group of a quandle
}

\author{
Agustín García Iglesias $^{1}$ - Leandro Vendramin ${ }^{2}$
}

Received: 31 January 2016 / Accepted: 6 September 2016

(C) Springer-Verlag Berlin Heidelberg 2016

\begin{abstract}
We use the inflation-restriction sequence and a result of Etingof and Graña on the rack cohomology to give a explicit description of 2-cocycles of finite indecomposable quandles with values in an abelian group. Several applications are given.
\end{abstract}

\section{Introduction and main results}

1.1. Quandles are non-associative algebraic structures introduced independently by Joyce [17] and Matveev [20] in connection with knot theory. They produce powerful invariants similar to those obtained by coloring [6,22]. Quandles turned out to be useful in different branches of algebra, topology and geometry since they have connections to several different topics such as permutation groups [16], quasigroups [24], symmetric spaces [25], Hopf algebras [2], etc.

Quandles have a very interesting cohomology theory that first appeared in [4] and independently in [12]. This theory is somewhat based on the rack cohomology introduced in Fenn et al. [11]. As in the case of groups, 2nd quandle cohomology groups can be used to produce new quandles by means of extensions.

This work is partially supported by CONICET, FONCyT PICT-2013-1414 and PICT-2014-1376, Secyt (UNC), ICTP and MATH-AmSud.

\footnotetext{
$凶$ Agustín García Iglesias

aigarcia@famaf.unc.edu.ar

Leandro Vendramin

lvendramin@dm.uba.ar

1 FaMAF-CIEM (CONICET), Universidad Nacional de Córdoba, Medina Allende s/n, Ciudad Universitaria (5000), Córdoba, Argentina

2 Departamento de Matemática-FCEN, Universidad de Buenos Aires, Pabellón I, Ciudad Universitaria (1428), Buenos Aires, Argentina
} 
The explicit computation of quandle cohomology groups is an important problem relevant to different areas of current research. The 2nd quandle cohomology group is particularly important since it has many applications going from knot theory to Hopf algebras.

In Carter et al. [4], used quandle cohomology classes to produce powerful invariants of classical links and their higher dimensional analogs. The invariants based on quandle 2cocycles improve the effectiveness of the quandle-coloring invariants since, for example, they distinguish knots from their mirror images. These invariants require an explicit description of 2-cocycles.

In the Hopf algebra context, quandles and their cohomology parametrize Yetter-Drinfeld modules. In turn these modules are crucial ingredients in the classification problem of finitedimensional Hopf algebras with non-abelian coradical. Indeed, an important step of the lifting method proposed by Andruskiewitsch and Schneider to solve this classification problem is the explicit computation of the 2nd cohomology of finite quandles, see [1].

1.2. In this work we give an explicit description of the second cohomology group of a finite indecomposable quandle. Our presentation is made by means of the characters of a certain finite group. This reduces the problem of computing 2-cocycles of a quandle to an easy manipulation involving cosets in a finite group. Our method is based on a result of Etingof and Graña [9] which relates the 2nd cohomology of a quandle and the first cohomology of an infinite group.

1.3. We now review the basics of our construction. Let $X$ be a finite quandle. Recall that the enveloping group of $X$ is the group

$$
G_{X}=\langle x \in X: x y=(x \triangleright y) x\rangle .
$$

Assume that $X$ is indecomposable and fix $x_{0} \in X$. Under the identification $\left\langle x_{0}\right\rangle \simeq \mathbb{Z}$ we show in Lemma 2.3 that $G_{X} \simeq N_{X} \rtimes \mathbb{Z}$, where $N_{X}$ is the commutator group [ $G_{X}, G_{X}$ ] of $G_{X}$. The group $G_{X}$ acts transitively on $X$ in a natural way, hence so does $N_{X}$, see Corollary 2.4. We denote by $N_{0}$ the stabilizer of $N_{X}$ on $x_{0}$ : this is a finite group $c f$. Lemma 2.1.

Fix an abelian group $A$ and let $M=\operatorname{Fun}(X, A)$ be the right $G_{X}$-module of functions $X \rightarrow A$, i.e. $(f \cdot x)(y)=f(x \triangleright y)$ for $x, y \in X$ and $f \in M$.

We prove that there is a commutative diagram with exact columns

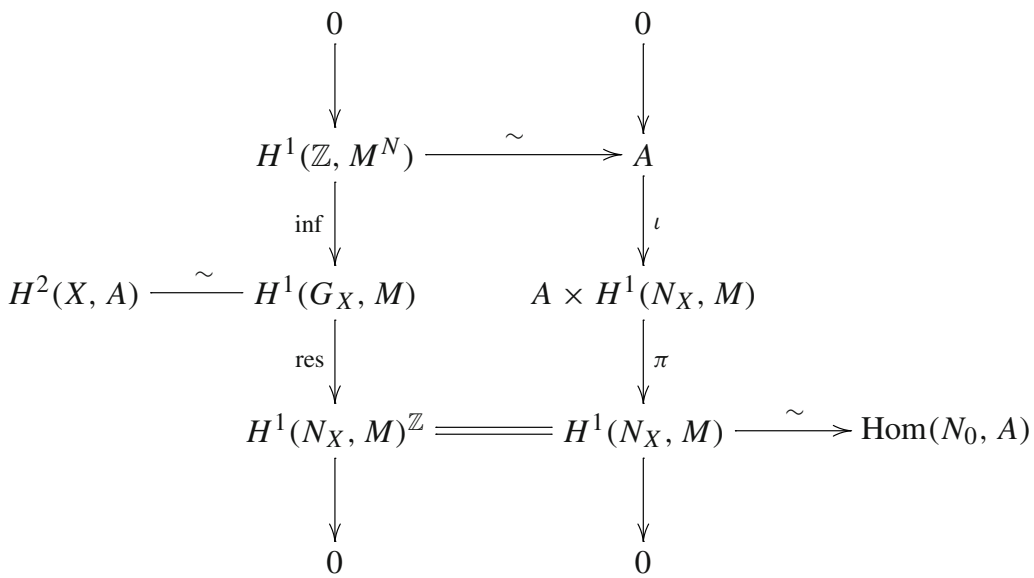


where the isomorphism

$$
H^{2}(X, A) \simeq H^{1}\left(G_{X}, M\right), \quad q \longmapsto f_{q},
$$

is [9, Corollary 5.4], see also (2.3); inf and res denote the inflation-restriction maps and $\iota$ and $\pi$ denote the canonical inclusion and projection.

See Lemmas 3.1, 3.4 and Proposition 3.7 for a proof of the isomorphisms and the equality in the rows of the diagram. The exactness of the first column is a well-known fact $c f$. Lemma 2.9. We show that it splits in Lemma 2.10.

By diagram chasing, we derive an isomorphism

$$
H^{2}(X, A) \simeq A \times \operatorname{Hom}\left(N_{0}, A\right) .
$$

From this isomorphism we obtain an explicit description of rack and quandle 2-cocycles with values in any abelian group $A$, see Theorem 1.1 .

We denote by $f \mapsto f_{0}$ the map $H^{1}\left(G_{X}, M\right) \rightarrow \operatorname{Hom}\left(N_{0}, A\right)$ deduced from the diagram above.

Our first main result reads as follows, see Sect. 3 for a proof.

Theorem 1.1 Let $X$ be a finite indecomposable quandle, $x_{0} \in X$ and $A$ an abelian group with trivial $G_{X}$-action. Then

$$
H^{2}(X, A) \simeq A \times \operatorname{Hom}\left(N_{0}, A\right), \quad q \mapsto\left(q_{x_{0}, x_{0}},\left(f_{q}\right)_{0}\right) .
$$

In particular this shows that the non-constant 2-cocycles on $X$ are controlled by a finite group.

1.4. Our second main result is a precise recipe to reconstruct a cocycle $q \in H^{2}(X, A)$ from a datum $(a, g) \in A \times \operatorname{Hom}\left(N_{0}, A\right)$. That is, we give a converse to the map in (1.3) to build all explicit 2-cocycles of a given quandle. To do this, we need to introduce some extra notation.

First, we fix a good coset decomposition

$$
N_{X}=\bigsqcup_{i=0}^{k} \sigma_{i} N_{0},
$$

into $N_{0}$-cosets, i.e. the representatives $\sigma_{0}, \ldots, \sigma_{k}$ are chosen so that:

(1) $\sigma_{0}=1$;

(2) for each $i \in\{0, \ldots, k\}$ there is $j \in\{0, \ldots, k\}$ such that $x_{0} \triangleright \sigma_{i}=\sigma_{j}$;

(3) for each $x \in X$ there is $j \in\{0, \ldots, k\}$ such that $\sigma_{j} \triangleright x_{0}=x$.

The existence of such a decomposition is given in Proposition 4.1, together with a recursive method for constructing it.

We define $\sigma: N \rightarrow\left\{\sigma_{0}, \ldots, \sigma_{k}\right\}, \sigma(n)=\sigma_{i}$ if $n \in \sigma_{i} N_{0}$. We set, $c f$. (3.4),

$$
c(n)=\sigma(n)^{-1} n \in N_{0} .
$$

Given $y \in X$ and $j \in\{0, \ldots, k\}$ such that $\sigma_{j} \triangleright x_{0}=y$ we write

$$
\sigma_{y}:=\sigma_{j}
$$

Our second main result is the following, see sect. 4 for the proof.

Theorem 1.2 Let $X$ be a finite indecomposable quandle, $x_{0} \in X$ and $A$ an abelian group with trivial $G_{X}$-action. Let $N_{X}=\bigsqcup_{i=0}^{k} \sigma_{i} N_{0}$ be a good decomposition of $N_{X}$ into $N_{0}$-cosets. For each $a \in A$ and $g \in \operatorname{Hom}\left(N_{0}, A\right)$, the map $q: X \times X \rightarrow A$ given by

$$
q_{x, y}=a+g\left(c\left(x \sigma_{y} x_{0}^{-1}\right)\right)
$$

is a 2-cocycle of $X$ with values in $A$. 
Combining Theorems 1.1, 1.2 and the isomorphism (1.2), namely

$$
q_{x, y}=f_{q}(x)(y), \quad q \in H^{2}(X, A),
$$

we immediately obtain the following corollary.

Corollary 1.3 Let $X$ be a finite indecomposable quandle, $x_{0} \in X$ and $A$ an abelian group with trivial $G_{X}$-action. Let $N_{X}=\bigsqcup_{i=0}^{k} \sigma_{i} N_{0}$ be a good decomposition of $N_{X}$ into $N_{0}$-cosets and let $q \in H^{2}(X, A)$. Then there exists $a \in A$ and $g \in \operatorname{Hom}\left(N_{0}, A\right)$ such that (1.4) holds for all $x, y \in X$.

Corollary 1.3 has many applications and can be used for explicit calculations of rack cohomology groups of quandles. In particular, if the commutator subgroup $N_{X}$ acts regularly on $X$, then $N_{0}=1$ and hence we obtain the following corollary.

Corollary 1.4 Let $X$ be a finite indecomposable quandle. If the action of $N_{X}$ on $X$ is regular, then $H^{2}\left(X, \mathbb{C}^{\times}\right) \simeq \mathbb{C}^{\times}$.

1.5. The paper is organized as follows. Preliminaries on racks and quandles, cohomology theory of groups, and cohomology theories of racks and quandles appear in Sect. 2. Our first main result, Theorem 1.1, is proved in Sect. 3. Theorem 1.2 is proved in Sect. 4. Applications of our theory are given in Sect. 5. These applications include the calculations of the 2 nd rack cohomology group of: (a) the quandle associated with the conjugacy class of transpositions, see Theorem 5.5; (b) affine racks of size $p$ and $p^{2}$, where $p$ is a prime number, see Propositions 5.8, 5.10, 5.11 and 5.12; and (c) another proof of Eisermann's formula for computing the 2nd quandle homology group of a quandle, see Theorem 5.6.

\section{Preliminaries}

\subsection{Notation}

For a set $X$ we denote by $\mathbb{S}_{X}$ the group of permutations $X \rightarrow X$. If $X$ is finite of cardinal $|X| \in \mathbb{N}$, then we identify $\mathbb{S}_{|X|}=\mathbb{S}_{X}$. For any group $G$ we denote by $[G, G]$ its commutator subgroup and $G_{\mathrm{ab}}$ its abelianization, i.e. $G_{\mathrm{ab}}=G /[G, G]$. In addition, $Z(G)$ is the center of $G$ and $G_{G}(g)=\{h \in G: h g=g h\}$ for $g \in G$. We denote by $\operatorname{Aut}(G)$ the group of automorphisms $G \rightarrow G$; if $\gamma \in \operatorname{Aut}(G)$, then $\operatorname{ord}(\gamma)$ is the order of $\gamma$.

Let $M$ be an abelian group equipped with a $G$-action. We denote by $H^{n}(G, M), n \geq 0$, the $n$th cohomology group of $G$ with coefficients on $M$. We denote by $Z^{n}(G, M)$, resp. $B^{n}(G, M)$, the groups of cocycles, resp. cobordisms, of $G$ with values on $M$. We refer the reader to [3] for unexplained notation and terminology.

\subsection{Racks}

A rack is a non-empty set $X$ together with a binary operation $\triangleright: X \times X \rightarrow X$ such that the maps $\varphi_{x}=x \triangleright-: X \rightarrow X, y \mapsto x \triangleright y$, are bijective for each $x \in X$, and $x \triangleright(y \triangleright z)=(x \triangleright y) \triangleright(x \triangleright z)$ for all $x, y, z \in X$. A quandle is a rack that further satisfies $x \triangleright x=x$ for all $x \in X$. A prototypical example of a rack is a group $G$ with $\triangleright$ given by conjugation. A rack is indecomposable if the inner group $\operatorname{Inn}(X)=\left\langle\varphi_{x}: x \in X\right\rangle \leq \mathbb{S}_{X}$ acts transitively on $X$. 
The enveloping group $G_{X} c f$. (1.1) also acts on $X$, and this action is readily seen to be transitive when $X$ is indecomposable. The group $G_{X}$ is infinite. There is a finite analogue of this group, which is constructed as follows: For each $x$, let $n_{x}=\operatorname{ord} \varphi_{x}$. Then the subgroup $Z_{X}=\left\langle x^{n_{x}}, x \in X\right\rangle \leq G_{X}$ is normal and the quotient $F_{X}=G_{X} / Z_{X}$ is finite, see [14, §2]. We write $N_{X}=\left[G_{X}, G_{X}\right]$ to denote the commutator subgroup of $G_{X}$.

Lemma 2.1 [15, Lemma 1.10] Let $X$ be an indecomposable quandle. Then $N_{X} \simeq\left[F_{X}, F_{X}\right]$. In particular, $N_{X}$ is finite.

The last claim of Lemma 2.1 also follows from the following result and a theorem of Schur, see for example [23, Theorem 5.32].

Lemma 2.2 Let $X$ be a finite indecomposable quandle. Then all conjugacy classes of $G_{X}$ are finite.

Proof Since $G_{X}$ acts transitively on $X$ and the center $Z\left(G_{X}\right)$ is the kernel of this action, it follows that the index $\left[G_{X}: Z\left(G_{X}\right)\right]$ is finite. This implies that all conjugacy classes of $G_{X}$ are finite as

$$
\left[G_{X}: C_{G_{X}}(g)\right] \leq\left[G_{X}: Z\left(G_{X}\right)\right],
$$

where $C_{G_{X}}(g)$ denotes the centralizer of $g$ in $G_{X}$.

We consider the unique surjective group homomorphism

$$
\mathrm{d}: G_{X} \rightarrow \mathbb{Z}
$$

satisfying $\mathrm{d}(x)=1$ for all $x \in X$. In particular, this homomorphism shows that $G_{X}$ is infinite and induces a notion of degree on $G_{X}$.

Lemma 2.3 Let $X$ be an indecomposable finite quandle and $x_{0} \in X$. Then the following hold:

(1) $G_{X}=\operatorname{kerd} \rtimes\left\langle x_{0}\right\rangle$.

(2) $\operatorname{ker} \mathrm{d}=N_{X}$ if $X$ is indecomposable.

Proof Since ker d is a normal subgroup of $G_{X}$, ker $\mathrm{d}\left\langle x_{0}\right\rangle$ is a subgroup of $G_{X}$. It is clear that ker $\mathrm{d} \cap\left\langle x_{0}\right\rangle=1$ comparing degrees. Finally $G_{X}=\operatorname{ker} \mathrm{d}\left\langle x_{0}\right\rangle$ since $x=\left(x x_{0}^{-1}\right) x_{0} \in \operatorname{ker} \mathrm{d}\left\langle x_{0}\right\rangle$ for all $x \in X$.

It is clear that $N_{X} \subseteq$ ker d. Next we prove the equality when $X$ is indecomposable. Let $\ell: G_{X} \rightarrow \mathbb{Z}$ be defined as $\ell(g)=n$, if $g=x_{i_{1}}^{\epsilon_{1}} \ldots x_{i_{n}}^{\epsilon_{n}}, \epsilon_{i} \in\{ \pm 1\}, i \in\{1, \ldots, n\}$, is a a reduced expression of $g$ in terms of the generators of $G_{X}$. We show that ker $\mathrm{d} \subseteq N_{X}$ by induction on $\ell(g), g \in \operatorname{ker}$. If $\ell(g)=2$, then $g=x_{i}^{ \pm 1} x_{j}^{\mp 1}$. So we may assume that $g=x_{i} x_{j}^{-1}$ (if not, take inverses). Now, as $X$ is indecomposable, there is $h \in G_{X}$ such that $h \cdot x_{j}=x_{i}$. Hence $g=h x_{j} h^{-1} x_{j}^{-1} \in N_{X}$. Now, if $\ell(g)>2$, then there is a reduced expression of $g$ (or $g^{-1}$ ) in which $g=g_{1} x_{i} x_{j}^{-1} g_{2}, x_{i}, x_{j} \in X$ and $g_{1}, g_{2} \in G_{X}$. Now, on the one hand, $0=\mathrm{d}(g)=\mathrm{d}\left(g_{1}\right)+\mathrm{d}\left(g_{2}\right)$ and thus $g_{1} g_{2} \in N_{X}$ as $\ell\left(g_{1} g_{2}\right)<\ell(g)$. On the other, $g=\left(g_{1} x_{i} x_{j}^{-1} g_{1}^{-1}\right)\left(g_{1} g_{2}\right)$ and therefore $g \in N_{X}$.

Corollary 2.4 The restriction of the action of $G_{X}$ on $X$ to $N_{X}$ is transitive.

Proof Let $x, y \in X$ and let $g \in G_{X}$ such that $g \cdot x=y$ and let $\ell=\mathrm{d}(g)$. Then $g^{\prime}=y^{-\ell} g \in$ $N_{X}$ by Lemma 2.3 and $g^{\prime} \cdot x=y$. 


\subsection{Rack cohomology}

A cohomology theory for racks was introduced in [10] and independently in [12]. A cohomology theory for quandles was developed in [4]. These theories were further developed and generalized for example in $[2,18]$.

We briefly recall these cohomology theories next. Let $X$ be a rack and let $M$ be a right $G_{X}$-module. Set $C^{n}=C^{n}(X, M)=\operatorname{Fun}\left(X^{n}, M\right), n \geq 0$, the set of functions from $X^{n}$ to $M$. Consider the differential $d: C^{n} \rightarrow C^{n+1}$

$$
\begin{aligned}
d f\left(x_{1}, \ldots, x_{n+1}\right)= & \sum_{i=1}^{n}(-1)^{i-1}\left(f\left(x_{1}, \ldots, x_{i-1}, x_{i+1}, \ldots, x_{n+1}\right)\right. \\
& \left.-f\left(x_{1}, \ldots, x_{i-1}, x_{i} \triangleright x_{i+1}, \ldots, x_{i} \triangleright x_{n+1}\right) \cdot x_{i}\right) .
\end{aligned}
$$

The rack cohomology $H^{\bullet}(X, M)$ of $X$ with coefficients in $M$ is the cohomology of the complex $\left(C^{\bullet}, d\right)$ [9, Definition 2.3]. The groups of cocycles resp. cobordisms, are denoted by $Z^{n}(X, M)$, resp. $B^{n}(G, M)$. When $A$ is an abelian group and no reference to a $G_{X}$-action on $A$ is specified, $H^{\bullet}(X, A)$ stands for the cohomology of $X$ with values in the trivial module $M=A$. If $q$ is a class in $H^{2}(X, A)$, we set $q_{x, y}:=q(x, y)$. Hence $q \in H^{2}(X, A)$ if and only if

$$
q_{x \triangleright y, x \triangleright z} q_{x, z}=q_{x, y \triangleright z} q_{y, z}, \quad \forall x, y, z \in X
$$

and two classes $q, q^{\prime} \in H^{2}(X, A)$ are equivalent if and only if there exists $\gamma: X \rightarrow A$ such that $q_{x, y}^{\prime}=q_{x, y} \gamma(x \triangleright y) \gamma(y)^{-1}$ for all $x, y \in X$.

The rack homology $H_{\bullet}(X, A)$ with values in an abelian group $A$ is defined analogously, by considering the free abelian group $F_{n}(X)$ on $X^{n}, n \geq 0$, and setting $C_{n}(X, A):=F_{n}(X) \otimes A$. If $X$ is a quandle, then the subgroup $F_{n}^{D}(X) \leq F_{n}(X)$ generated by $n$-tuples $\left(x_{1}, \ldots x_{n}\right)$ with $x_{i}=x_{i+1}$ for some $i$, defines a subcomplex $C_{\bullet}^{D}=C_{\bullet}^{D}(X, A)$ of $C_{\bullet}$. The quandle homology $H_{\bullet}^{Q}(X, A)$ of $X$ is the homology of the quotient complex $C_{\bullet}^{Q}=\left(C_{n} / C_{n}^{D}\right)_{n \geq 0}$.

In this work we give a description of the group $H^{2}(X, A)$ of 2-cocycles on $X$ with values in an abelian group $A$, which allows us to compute cocycles explicitly. We recall next some identifications between the (co)homology theories described above that will be useful for our goal.

Lemma 2.5 [5, Proposition 3.4] $H^{2}(X, A) \simeq \operatorname{Hom}\left(H_{2}(X, \mathbb{Z}), A\right)$, via

$$
H^{2}(X, A) \ni q \mapsto\left([x, y] \mapsto q_{x, y}\right) \in \operatorname{Hom}\left(H_{2}(X, \mathbb{Z}), A\right) .
$$

The following is a particular case of [19, Theorem 7].

Lemma 2.6 Assume $X$ is an indecomposable quandle. Then

$$
H_{2}(X, \mathbb{Z}) \simeq H_{2}^{Q}(X, \mathbb{Z}) \times \mathbb{Z} .
$$

Explicitly, if $(x, y) \in X^{2}$, then the isomorphism is induced by the map

$$
(x, y) \mapsto \begin{cases}(x, y) \times 0, & \text { if } x \neq y \\ 0 \times 1, & \text { if } x=y .\end{cases}
$$

Etingof and Graña found a deep relation between group cohomology and rack cohomology. 
Theorem 2.7 [9, Corollary 5.4] Let $X$ be a finite indecomposable rack and A an abelian group with a trivial $G_{X}$-action. Then

$$
H^{1}\left(G_{X}, \operatorname{Fun}(X, A)\right) \simeq H^{2}(X, A) .
$$

This equivalence is given as follows:

(1) If $f \in H^{1}(G, \operatorname{Fun}(X, A))$ then a 2-cocycle $q^{f} \in H^{2}(X, A)$ arises as

$$
q_{x, y}^{f}=f(x)(y), \quad x, y \in X .
$$

(2) Conversely, $q \in H^{2}(X, A)$ determines $f_{q} \in H^{1}(G, \operatorname{Fun}(X, A))$ by extending $q$ recursively via

$$
f_{q}(x y)(z)=q_{x, y \triangleright z}+q_{y, z}, \quad x, y, z \in X .
$$

Remark 2.8 Let $G$ be a (non-abelian) group and fix $Z^{2}(X, G) \subset \operatorname{Fun}\left(X^{2}, G\right)$ as the subset of all $q: X^{2} \rightarrow G$ satisfying (2.2). We say that $q$ is equivalent to $q^{\prime}$, and we write $q \sim q^{\prime}$, in $Z^{2}(X, G)$ if and only if there is $\gamma \in \operatorname{Fun}(X, G)$ such that $q_{x, y}^{\prime}=\gamma(x \triangleright y) q_{x, y} \gamma(y)^{-1}$. If $H^{2}(X, G):=Z^{2}(X, G) / \sim$, then Theorem 2.7 holds, see [9, Remark 5.6].

\subsection{Group cohomology}

Let $G$ be a group, $N \triangleleft G$ a normal subgroup and $M$ a right $G$-module. Recall $c f$. [3, 3.8] that there is a right $G / N$-action on $H^{1}(N, M)$, induced by

$$
(f \cdot g)(n)=f\left(g n g^{-1}\right) \cdot g, \quad g \in G, n \in N, f \in H^{1}(N, M) .
$$

Indeed, let $f \in Z^{1}(N, M)$. If $g \in N$, then

$$
(f \cdot g)(n)=f(g n)-f(g)=f(g) \cdot n+f(n)-f(g)
$$

by the cocycle condition. Hence

$$
(f \cdot g)(n)-f(n)=f(g) \cdot n-f(g)
$$

and thus $f \cdot g=f \in H^{1}(N, M)$. The inflation-restriction sequence is

$$
\begin{aligned}
0 \rightarrow H^{1}\left(G / N, M^{N}\right) & \stackrel{\iota}{\rightarrow} H^{1}(G, M) \stackrel{r}{\rightarrow} H^{1}(N, M)^{G / N} \\
& \rightarrow H^{2}\left(G / N, M^{N}\right) \rightarrow H^{2}(G, M)
\end{aligned}
$$

where the inflation map $\iota(h), h \in H^{1}\left(G / N, M^{N}\right)$, is the composition

$$
G \rightarrow G / N \stackrel{h}{\rightarrow} M^{N} \hookrightarrow M
$$

and the restriction map $r(g), g \in H^{1}(G, M)$, is the composition

$$
N \hookrightarrow G \stackrel{g}{\rightarrow} M .
$$

In the case where $G / N \simeq \mathbb{Z}$ one obtains the following result, see loc.cit.

Lemma 2.9 Assume that $G / N \simeq \mathbb{Z}$. Then

(1) $H^{2}\left(G / N, M^{N}\right)=0$.

(2) $H^{1}\left(G / N, M^{N}\right)=M^{N} /\langle m \cdot g-m\rangle$, (class of) $f \mapsto$ (class of) $f(1)$. 
In particular, the exact sequence (2.5) reduces to

$$
0 \rightarrow H^{1}\left(G / N, M^{N}\right) \stackrel{\inf }{\rightarrow} H^{1}(G, M) \stackrel{\text { res }}{\rightarrow} H^{1}(N, M)^{G / N} \rightarrow 0 .
$$

Lemma 2.10 Assume that $N$ is finite and $G / N \simeq \mathbb{Z}$. Then (2.6) splits. A retraction for inf : $H^{1}\left(G / N, M^{N}\right) \rightarrow H^{1}(G, M)$ is given by

$$
j: H^{1}(G, M) \rightarrow H^{1}\left(G / N, M^{N}\right), \quad j(f)(\ell)=\frac{1}{|N|} \sum_{n \in N}\left(f\left(n x_{0}^{\ell}\right)-f(n)\right) .
$$

Proof We need to check that $j$ is well-defined, that is:

(1) If $f \in Z^{1}(G, M)$, then $j(f)(G / N) \subseteq M^{N}$.

(2) If $f \in Z^{1}(G, M)$, then $j(f) \in Z^{1}\left(G / N, M^{N}\right)$.

(3) If $f \in B^{1}(G, M)$, then $j(f) \in B^{1}\left(G / N, M^{N}\right)$.

Let $f \in Z^{1}(G, M)$ and set $\varphi:=j(f)$. For (1), using the cocycle condition,

$$
\begin{aligned}
\varphi(\ell) \cdot n & =\frac{1}{|N|} \sum_{m \in N}\left(f\left(m x_{0}^{\ell}\right) \cdot n-f(m) \cdot n\right) \\
& =\frac{1}{|N|} \sum_{m \in N}\left(f\left(m x_{0}^{\ell} n\right)-f(n)-f(m n)+f(n)\right) \\
& =\frac{1}{|N|} \sum_{m \in N}\left(f\left(m x_{0}^{\ell} n x_{0}^{-\ell} x_{0}^{\ell}\right)-f(m n)\right) .
\end{aligned}
$$

By reordering the sum, $\varphi(\ell) \cdot n=\varphi(\ell)$ for all $n \in N, \ell \in \mathbb{Z}$. Hence (1) holds.

In (2), we get

$$
\begin{aligned}
\varphi(\ell+r) & =\frac{1}{|N|} \sum_{n \in N}\left(f\left(n x_{0}^{\ell+r}\right)-f(n)\right) \\
& =\frac{1}{|N|} \sum_{n \in N}\left(f\left(n x_{0}^{\ell}\right) \cdot x_{0}^{r}+f\left(x_{0}^{r}\right)-f(n)\right) \\
& =\frac{1}{|N|} \sum_{n \in N}\left(f\left(n x_{0}^{\ell}\right) \cdot x_{0}^{r}-f(n) \cdot x_{0}^{r}+f(n) \cdot x_{0}^{r}+f\left(x_{0}^{r}\right)-f(n)\right) \\
& =\varphi(\ell) \cdot r+\frac{1}{|N|} \sum_{n \in N}\left(f\left(n x_{0}^{r}\right)-f\left(x_{0}^{r}\right)+f\left(x_{0}^{r}\right)-f(n)\right) \\
& =\varphi(\ell) \cdot r+\varphi(r) .
\end{aligned}
$$

Thus (2) holds. If $f \in B^{1}(G, M)$, then there exists $\psi \in M$ such that $f(g)=\psi \cdot g-\psi$. Hence,

$$
\begin{aligned}
j(f)(\ell) & =\frac{1}{|N|} \sum_{n \in N}\left(\psi \cdot n x_{0}^{\ell}-\psi-\psi \cdot n+\psi\right) \\
& =\frac{1}{|N|} \sum_{n \in N}\left(\psi \cdot n x_{0}^{\ell}-\psi \cdot n\right)
\end{aligned}
$$

and thus $j(f)(\ell)=\gamma \cdot \ell-\gamma$ for

$$
\gamma=\frac{1}{|N|} \sum_{n \in N} \psi \cdot n \in M^{N}
$$


This shows (3). Finally we prove that $j \circ$ inf $=$ id. For this, recall that if $\varphi \in H^{1}\left(G / N, M^{N}\right)$ and $g \in G$, then $\inf (\varphi)(g)=\varphi(\bar{g})$, where $\bar{g}$ is the class of $g$ in $G / N \simeq \mathbb{Z}$. Then

$$
(j \circ \inf )(\varphi)(\ell)=\frac{1}{|N|} \sum_{n \in N}\left(\inf (\varphi)\left(n x_{0}^{\ell}\right)-\inf (\varphi)(n)\right)=\frac{1}{|N|} \sum_{n \in N} \varphi(\ell)=\varphi(\ell)
$$

for all $\ell \in \mathbb{Z}$. This completes the proof.

\section{Proof of Theorem 1.1}

Assume that $X$ is a finite indecomposable rack. We write $G=G_{X}, N=\left[G_{X}, G_{X}\right]$. Let $A$ be an abelian group with trivial $G$-action and set $M=\operatorname{Fun}(X, A)$. Fix $x_{0} \in X$ and $G \simeq N \rtimes \mathbb{Z}$ as in Lemma 2.3. It follows from Lemma 2.10 that

$$
0 \rightarrow H^{1}\left(G / N, M^{N}\right) \stackrel{\text { inf }}{\longrightarrow} H^{1}(G, M) \stackrel{\text { res }}{\longrightarrow} H^{1}(N, M)^{G / N} \rightarrow 0
$$

splits. We first identify the first term of this sequence.

Lemma 3.1 $H^{1}\left(\mathbb{Z}, M^{N}\right) \simeq A$, via $f \mapsto f(1)\left(x_{0}\right)$.

Proof Recall from Lemma 2.9(2) that $H^{1}\left(\mathbb{Z}, M^{N}\right) \simeq M^{N} / F$, where $F$ is the submodule generated by $\left\{\varphi \cdot x_{0}^{p}-\varphi: p \in \mathbb{Z}, \varphi \in M^{N}\right\}$. Since $X=N \triangleright\left\{x_{0}\right\}$ by Corollary 2.4 and $n \triangleright x_{0}=x \in X$ for some $n \in N$,

$$
\varphi(x)=\varphi\left(n \triangleright x_{0}\right)=(\varphi \cdot n)\left(x_{0}\right)
$$

for all $\varphi \in M$. Hence, if $\varphi \in M^{N}$, then $\varphi(x)=\varphi\left(x_{0}\right), x \in X$. Consequently, $F=\{0\}$ and $H^{1}\left(\mathbb{Z}, M^{N}\right) \simeq M^{N}$. But $M^{N} \simeq A$ as any $\varphi \in M^{N}$ is determined by its value $\varphi\left(x_{0}\right) \in A$. Hence the lemma follows.

As for the third term, we will show in Proposition 3.7 that

$$
H^{1}(N, M)^{\mathbb{Z}} \simeq \operatorname{Hom}\left(N_{0}, A\right) .
$$

To do so, we first need several lemmas.

Lemma 3.2 The map

$$
Z^{1}(N, M) \rightarrow \operatorname{Hom}\left(N_{0}, A\right), \quad f \mapsto f_{0},
$$

where $f_{0}\left(n_{0}\right)=f\left(n_{0}\right)\left(x_{0}\right)$ for $n_{0} \in N_{0}$, is well-defined and factors to a map $H^{1}(N, M) \rightarrow$ $\operatorname{Hom}\left(N_{0}, A\right)$.

Proof We first prove that $f_{0}$ is indeed a group homomorphism:

$$
\begin{aligned}
f_{0}\left(n_{0} n_{0}^{\prime}\right) & =f\left(n_{0} n_{0}^{\prime}\right)\left(x_{0}\right)=\left(f\left(n_{0}\right) \cdot n_{0}^{\prime}\right)\left(x_{0}\right)+f\left(n_{0}^{\prime}\right)\left(x_{0}\right) \\
& =f\left(n_{0}\right)\left(n_{0}^{\prime} \triangleright x_{0}\right)+f\left(n_{0}^{\prime}\right)\left(x_{0}\right) \\
& =f\left(n_{0}\right)\left(x_{0}\right)+f\left(n_{0}^{\prime}\right)\left(x_{0}\right)=f_{0}\left(n_{0}\right)+f_{0}\left(n_{0}^{\prime}\right), n_{0}, n_{0}^{\prime} \in N_{0} .
\end{aligned}
$$

We now show that the map factors to a map $H^{1}(N, M) \rightarrow \operatorname{Hom}\left(N_{0}, A\right)$. Let $f \in$ $B^{1}(N, M)$, that is $f(n)=\varphi \cdot n-\varphi$ for some $\varphi \in M$. Then

$$
\begin{aligned}
f_{0}\left(n_{0}\right) & =f\left(n_{0}\right)\left(x_{0}\right)=\left(\varphi \cdot n_{0}\right)\left(x_{0}\right)-\varphi\left(x_{0}\right) \\
& =\varphi\left(n_{0} \triangleright x_{0}\right)-\varphi\left(x_{0}\right)=\varphi\left(x_{0}\right)-\varphi\left(x_{0}\right)=0 .
\end{aligned}
$$

This completes the proof. 
Lemma 3.3 The map $H^{1}(N, M) \rightarrow \operatorname{Hom}\left(N_{0}, A\right), f \mapsto f_{0}$, is an injective group homomorphism.

Proof It is clear that $f \mapsto f_{0}$ is a group homomorphism.

Let $f \in H^{1}(N, M)$ be such that $f_{0}=0$. That is, $f\left(n_{0}\right)\left(x_{0}\right)=0$ for every $n_{0} \in N_{0}$. We claim that there is $\varphi \in M$ such that $f(m)=(\varphi \cdot m)-\varphi$ and thus $f=0$ in $H^{1}(N, M)$. Set

$$
\varphi(x):=f(n)\left(x_{0}\right) \quad \text { if } x=n \triangleright x_{0} .
$$

Let us check that this is well-defined: if $x=n \triangleright x_{0}=n^{\prime} \triangleright x_{0}$, then $n^{-1} n^{\prime} \in N_{0}$. Since $f(1)=0$, one obtains that $f\left(n^{-1}\right)=-f(n) \cdot n^{-1}$. Then

$$
\begin{aligned}
0 & =f_{0}\left(n^{-1} n^{\prime}\right)=f\left(n^{-1} n^{\prime}\right)\left(x_{0}\right) \\
& =-f(n)\left(n^{-1} n^{\prime} \triangleright x_{0}\right)+f\left(n^{\prime}\right)\left(x_{0}\right)=-f(n)\left(x_{0}\right)+f\left(n^{\prime}\right)\left(x_{0}\right),
\end{aligned}
$$

and thus $\varphi(x)$ does not depend on $n \in N$ such that $x=n \triangleright x_{0}$. Finally for each $m \in N$ and every $x=n \triangleright x_{0} \in X$ with $n \in N$,

$$
\begin{aligned}
& (\varphi \cdot m-\varphi)(x)=\varphi(m \triangleright x)-\varphi(x)=\varphi\left(m \triangleright n \triangleright x_{0}\right)-\varphi\left(n \triangleright x_{0}\right) \\
& \quad=f(m n)\left(x_{0}\right)-f(n)\left(x_{0}\right)=(f(m) \cdot n)\left(x_{0}\right)+f(n)\left(x_{0}\right)-f(n)\left(x_{0}\right) \\
& \quad=f(m)\left(n \triangleright x_{0}\right)=f(m)(x),
\end{aligned}
$$

and therefore $f=0$.

Recall the definition of the $\mathbb{Z}$-action on $H^{1}(N, M)$ from (2.4).

Lemma 3.4 Assume $X$ is a quandle. Then $H^{1}(N, M)=H^{1}(N, M)^{\mathbb{Z}}$.

Proof Let $f \in H^{1}(N, M)$ and set $g=f-f \cdot x_{0}$. If $n_{0} \in N_{0}$, then

$$
g_{0}\left(n_{0}\right)=f\left(n_{0}\right)\left(x_{0}\right)-f\left(x_{0} n_{0} x_{0}^{-1}\right)\left(x_{0} \triangleright x_{0}\right)=0 .
$$

Thus $g_{0}=0$ and hence $f=f \cdot x_{0}$ for all $f \in H^{1}(N, M)$ by Lemma 3.3, since the group homomorphism $g \mapsto g_{0}$ is injective.

In order to show the surjectivity of the map $f \mapsto f_{0}$ from Lemma 3.3, we need to fix a decomposition of $N$ into $N_{0}$-cosets

$$
N=\bigsqcup_{i=0}^{k} \sigma_{i} N_{0},
$$

where $\sigma_{i} \in N$ is a representative, $\sigma_{0} N_{0}=N_{0}$. We define

$$
\sigma: N \rightarrow\left\{\sigma_{0}, \ldots, \sigma_{k}\right\}, \quad \sigma(n)=\sigma_{i} \quad \text { if } n \in \sigma_{i} N_{0} .
$$

For $n \in N$ we consider $c(n) \in N_{0}$ defined by

$$
n=\sigma(n) c(n) \text {. }
$$

Remark 3.5 For all $n \in N$ and $n_{0} \in N_{0}$ it follows that $c\left(n n_{0}\right)=c(n) n_{0}$. Indeed, $n n_{0}=$ $\sigma(n) c(n) n_{0}=\sigma\left(n n_{0}\right) c\left(n n_{0}\right)$ and thus the claim holds since each $m \in N$ decomposes uniquely as $m=\sigma(m) c(m)$.

Lemma 3.6 The map $H^{1}(N, M) \rightarrow \operatorname{Hom}\left(N_{0}, A\right), f \mapsto f_{0}$, is surjective. 
Proof Let $g: N_{0} \rightarrow A$ be a group homomorphism; we shall construct an $f \in Z^{1}(N, M)$ such that $f_{0}=g$. We claim that the map $f: N \rightarrow M, n \mapsto f(n)$, given by

$$
f(n)(x)=g(c(n m))-g(c(m))=g\left(c(n m) c(m)^{-1}\right),
$$

where $m \in N$ is such that $x=m \triangleright x_{0}$, is well-defined. Indeed, if $m^{\prime} \in N$ also satisfies $x=m^{\prime} \triangleright x_{0}$, then $m^{-1} m^{\prime} \in N_{0}$ and thus $\sigma(m)^{-1} \sigma\left(m^{\prime}\right) \in N_{0}$. That is $\sigma(m)=\sigma\left(m^{\prime}\right)$ and thus $\sigma(n m)=\sigma\left(n m^{\prime}\right)$ for every $n \in N$ since $\left(n m^{\prime}\right)^{-1} n m \in N_{0}$. As $g$ is a group homomorphism,

$$
g\left(c(n m) c(m)^{-1}\right)-g\left(c\left(n m^{\prime}\right) c\left(m^{\prime}\right)^{-1}\right)=g\left(c(n m) c(m)^{-1} c\left(m^{\prime}\right) c\left(n m^{\prime}\right)^{-1}\right) .
$$

Now, $c(n m) c(m)^{-1} c\left(m^{\prime}\right) c\left(n m^{\prime}\right)^{-1}$ is, by definition,

$$
\left(\sigma(n m)^{-1} n m\right)\left(m^{-1} \sigma(m)\right)\left(\sigma\left(m^{\prime}\right)^{-1} m^{\prime}\right)\left(m^{\prime-1} n^{-1} \sigma\left(n m^{\prime}\right)\right)=1 .
$$

Hence $g\left(c(n m) c(m)^{-1}\right)-g\left(c\left(n m^{\prime}\right) c\left(m^{\prime}\right)^{-1}\right)=g(1)=0$ and thus $f$ does not depend on the choice of $m$.

Now we show that $f \in Z^{1}(N, M)$. Let $x \in X, n, n^{\prime} \in N$ and $m \in N$ be such that $x=m \triangleright x_{0}$. On the one hand, we have

$$
f\left(n n^{\prime}\right)(x)=g\left(c\left(n n^{\prime} m\right)\right)-g(c(m)) .
$$

On the other,

$$
\begin{aligned}
\left(f(n) \cdot n^{\prime}\right)(x)+f\left(n^{\prime}\right)(x) & =f(n)\left(n^{\prime} \triangleright x\right)+f\left(n^{\prime}\right)(x) \\
& =g\left(c\left(n n^{\prime} m\right)\right)-g\left(c\left(n^{\prime} m\right)\right)+g\left(c\left(n^{\prime} m\right)\right)-g(c(m)) \\
& =f\left(n n^{\prime}\right)(x) .
\end{aligned}
$$

Finally we see that $g=f_{0}$, that is $f_{0}(n)=g(n)$ for $n \in N_{0}$. Now, if $n \in N_{0}$, then $c(n)=c(1 \cdot n)=c(1) n c f$. Remark 3.5. Also, as as $x_{0}=1 \triangleright x_{0}$,

$$
\begin{aligned}
f_{0}(n) & =f(n)\left(x_{0}\right)=g\left(c(n \cdot 1) c(1)^{-1}\right)=g(c(1 \cdot n))-g(c(1)) \\
& =g(c(1) n)-g(c(1))=g(c(1))+g(n)-g(c(1))=g(n)
\end{aligned}
$$

and the lemma follows.

Now we proceed to show (3.1).

Proposition 3.7 The map $Z^{1}(N, M) \rightarrow \operatorname{Hom}\left(N_{0}, A\right)$ given by $f \mapsto f_{0}$, where $f_{0}\left(n_{0}\right)=$ $f\left(n_{0}\right)\left(x_{0}\right)$ for $n_{0} \in N_{0}$, induces a group isomorphism

$$
H^{1}(N, M)^{\mathbb{Z}} \rightarrow \operatorname{Hom}\left(N_{0}, A\right)
$$

Proof Lemma 3.4 implies that $H^{1}(N, M)^{\mathbb{Z}} \simeq H^{1}(N, M)$ and Lemmas 3.3 and 3.6 yield $H^{1}(N, M) \simeq \operatorname{Hom}\left(N_{0}, A\right)$, as desired.

This allows us to complete the proof of Theorem 1.1.

Proof of Theorem 1.1 Using the cocycle condition, we get

$$
\begin{aligned}
j(f)(\ell) & =\frac{1}{|N|} \sum_{n \in N}\left(f(n) \cdot x_{0}^{\ell}+f\left(x_{0}^{\ell}\right)-f(n)\right) \\
& =f\left(x_{0}^{\ell}\right)+\frac{1}{|N|} \sum_{n \in N}\left(f(n) \cdot x_{0}^{\ell}-f(n)\right) .
\end{aligned}
$$


Hence, as $X$ is a quandle, for each $\ell \in \mathbb{Z}$,

$$
j(f)(\ell)\left(x_{0}\right)=f\left(x_{0}\right)\left(x_{0}\right) .
$$

Since $H^{1}\left(G / N, M^{N}\right) \simeq A$ by Lemma 3.1 and by Proposition 3.7 there exists an isomorphism $\zeta: H^{1}(N, M)^{\mathbb{Z}} \simeq \operatorname{Hom}\left(N_{0}, A\right)$, we write the inflation-restriction sequence (2.6) as

$$
0 \rightarrow A \stackrel{\inf _{0}}{\longrightarrow} H^{1}(G, M) \stackrel{\text { res }_{0}}{\longrightarrow} \operatorname{Hom}\left(N_{0}, A\right) \rightarrow 0,
$$

where $\operatorname{res}_{0}(f)=\operatorname{res}(f)_{0}$ for all $f \in H^{1}(G, M)$ and $\inf _{0}$ is the composition $A \simeq M^{N} \simeq$ $H^{1}\left(G / N, M^{N}\right)$. We set $f_{0}=\operatorname{res}_{0}(f)$ by abuse of notation, i.e.

$$
f_{0}\left(n_{0}\right)=f\left(n_{0}\right)\left(x_{0}\right), \quad n_{0} \in N_{0} .
$$

A retraction for $\inf _{0}$ is given by the composition

$$
j_{0}: H^{1}(G, M) \stackrel{j}{\rightarrow} H^{1}\left(G / N, M^{N}\right) \simeq A,
$$

using Lemmas 2.10 and 3.1, that is

$$
j_{0}(f)=j(f)(1)\left(x_{0}\right)=f\left(x_{0}\right)\left(x_{0}\right),
$$

cf. (3.6). Hence $H^{1}(G, M) \simeq A \times \operatorname{Hom}\left(N_{0}, A\right)$ via

$$
f \mapsto\left(f\left(x_{0}\right)\left(x_{0}\right), f_{0}\right) .
$$

This completes the proof.

\section{Proof of Theorem 1.2}

In this section we show the Reconstruction Theorem 1.2. We fix $x_{0} \in X$ and write $N_{0} \leq N_{X}$ for the stabilizer of $x_{0}$ in $N_{X}$. By Lemma 2.1, $N_{0}$ is a finite group.

The key for the proof of Theorem 1.2 lays in the existence of a particular class of decompositions

$$
N_{X}=\bigsqcup_{i=0}^{k} \sigma_{i} N_{0}
$$

of $N_{X}$ into $N_{0}$-cosets, which are good in our context.

Proposition 4.1 Let $X$ be a finite indecomposable quandle. Then there exists a decomposition $N_{X}=\bigsqcup_{i=0}^{k} \sigma_{i} N_{0}$ of $N_{X}$ into $N_{0}$-cosets such that the following hold:

(1) $\sigma_{0}=1$

(2) For each $i \in\{0, \ldots, k\}$ there is $j \in\{0, \ldots, k\}$ such that $x_{0} \triangleright \sigma_{i}=\sigma_{j}$.

(3) For each $x \in X$ there is $j \in\{0, \ldots, k\}$ such that $\sigma_{j} \triangleright x_{0}=x$.

Proof Fix a decomposition into cosets $N_{X}=\bigsqcup_{i=0}^{k} \sigma_{i} N_{0}$. Recall from (3.3) and (3.4) the definition of the corresponding assignments

$$
\sigma: N \rightarrow\left\{\sigma_{0}, \ldots, \sigma_{k}\right\} \text { and } c: N \rightarrow N_{0}
$$


Since $\sigma_{0}=1$, (1) holds. Condition (3) also holds trivially: If $x \in X$, there is $n \in N_{X}$ is such $n \triangleright x_{0}=x$ by Corollary 2.4. Now, there is $j \in\{0, \ldots, k\}$ such that $n \in \sigma_{j} N_{0}$, that is $n=\sigma_{j} n_{0}$ for some $n_{0} \in N_{0}$. Then $x=n \triangleright x_{0}=\sigma_{j} \triangleright\left(n_{0} \triangleright x_{0}\right)=\sigma_{j} \triangleright x_{0}$.

For Condition (2), set $S=\left\{\sigma_{1}, \ldots, \sigma_{k}\right\}$. We define

$$
t_{j}=t_{j}(S):=\min \left\{t \geq 1: x_{0}^{t} \triangleright \sigma_{j}=\sigma_{j}\right\}
$$

for all $j \in\{1, \ldots, k\}$. Observe that $1 \leq t_{j}(S) \leq \operatorname{ord} \varphi_{x_{0}}$, cf. $\$ 2.2$. For $i \in\{1, \ldots, k\}$ and $t \in\left\{0, \ldots, t_{j}(S)-1\right\}$ we define $\tau_{j, t}=x_{0}^{t} \triangleright \sigma_{j}$ and let

$$
T=\left\{\tau_{j, t}: 1 \leq j \leq k, 1 \leq t<t_{j}(S)\right\} .
$$

It is clear that $S \subseteq T$, as $\sigma_{j}=\tau_{j, 0}$ by definition, and that if $S=T$, then we are done. Notice that this is not a multi-set: we may have $\tau_{j, t}=\tau_{j^{\prime}, t^{\prime}}$, for different $(j, t),\left(j^{\prime}, t^{\prime}\right)$. On the other hand, if $t \neq t^{\prime}$, then $\tau_{j, t} \neq \tau_{j, t^{\prime}}$ for every $j$, since $t<t_{j}(S)$. In other words, there are $r \leq k$, $1=i_{1}<i_{2}<\cdots<i_{r}$ and $s_{j} \leq t_{i_{j}}, 1 \leq j \leq r$ such that

$$
T=\left\{\tau_{i_{j}, t}: 1 \leq j \leq r, 1 \leq t<s_{j}\right\}
$$

and $\tau_{i_{j}, t} \neq \tau_{i_{j^{\prime}}, t^{\prime}}$ if $j \neq j^{\prime}$ or $t \neq t^{\prime}$. We reorder the set $S$ so $i_{j}=j, j=1, \ldots, r$. If $S \neq T$, then we proceed inductively: we order $T$ by:

$$
\tau_{i, s} \prec \tau_{j, t} \Longleftrightarrow i<j \text { or } i=j \text { and } s<t .
$$

Let $\tau=\min \left\{\tau_{j, t}: \tau_{j, t} \notin S\right\}$ and let $\ell$ be such that $\sigma(\tau)=\sigma_{\ell}$, i.e. $\tau_{j, t}=x_{0}^{t} \triangleright \sigma_{j} \in \sigma_{\ell} N$ and $\tau_{j, t} \neq \sigma_{\ell}$. Observe that if $\tau=\tau_{j, t}$, then $\ell \neq j$. Set $S_{0}=S$ and $T_{0}=T$. We make a new choice of representatives replacing the original set $S_{0}$ by

$$
S_{1}=\left(S_{0} \backslash\left\{\sigma_{\ell}\right\}\right) \cup\{\tau\}=\left\{\sigma_{1}, \ldots, \sigma_{\ell-1}, \tau, \sigma_{\ell+1}, \ldots, \sigma_{k}\right\} .
$$

Define $t_{j}\left(S_{1}\right)$ and $\left(T_{1}, \prec\right)$ accordingly. We claim that $t_{j}\left(S_{1}\right) \leq t_{j}\left(S_{0}\right)$ for all $j$. Indeed, equality holds if $j \neq \ell$ and it readily follows that

$$
t_{\ell}\left(S_{1}\right)=t_{\ell}\left(S_{0}\right)-t<t_{\ell}\left(S_{0}\right)
$$

In particular, it follows that $|S|=\left|S_{1}\right| \leq\left|T_{1}\right|<\left|T_{0}\right|$. (This also follows as when constructing $T_{1}$ we are removing all the $\tau_{\ell, t}$.) If $T_{1}=S_{1}$, then we are done. Otherwise, we repeat this procedure until we end up with $S_{p}=T_{p}$ for some $p>1$. Then $S_{p}$ becomes the set of representatives we searched for.

We say that a decomposition of $N_{X}$ into $N_{0}$-cosets satisfying the conditions in Proposition 4.1 is good.

If $N_{X}=\bigsqcup_{i=0}^{k} \sigma_{i} N_{0}$ is a good decomposition, then for each $y \in X$ we set

$$
\sigma_{y}:=\sigma_{j}
$$

for $j \in\{0, \ldots, k\}$ such that $\sigma_{j} \triangleright x_{0}=y$.

Lemma 4.2 If $N_{X}=\bigsqcup_{i=0}^{k} \sigma_{i} N_{0}$ is good, then

$$
c\left(x_{0} \triangleright n\right)=c(n) .
$$

Proof Indeed, $x_{0} \triangleright n=x_{0} \sigma(n) x_{0}^{-1} c(n)$, as $c(n) \in N_{0}$ and $x_{0} \sigma(n) x_{0}^{-1}=\sigma_{i}$, for some $i \in\{0, \ldots, k\}$.

Recall the definition of the group homomorphism $d: G_{X} \rightarrow \mathbb{Z}$ from (2.1). 
Lemma 4.3 For each $u \in G_{X}$ and $y \in X$,

$$
\sigma_{u \triangleright y}=u \sigma_{y} x_{0}^{-\mathrm{d}(u)} c\left(u \sigma_{y} x_{0}^{-\mathrm{d}(u)}\right)^{-1} .
$$

In particular if $n \in N$, then $\sigma_{n \triangleright y}=\sigma\left(n \sigma_{y}\right)$.

Proof Since

$$
\sigma_{u \triangleright y} \triangleright x_{0}=u \triangleright y=u \triangleright\left(\sigma_{y} \triangleright x_{0}\right)=\left(u \sigma_{y}\right) \triangleright x_{0}=\left(u \sigma_{y} x_{0}^{-\mathrm{d}(u)}\right) \triangleright x_{0}
$$

and $u \sigma_{y} x_{0}^{-\mathrm{d}(u)} \in N$, it follows that $\sigma_{u \triangleright y}=\sigma\left(u \sigma_{y} x_{0}^{-\mathrm{d}(u)}\right)$. Then

$$
u \sigma_{y} x_{0}^{-\mathrm{d}(u)}=\sigma_{u \triangleright y} c\left(u \sigma_{y} x_{0}^{-\mathrm{d}(u)}\right),
$$

and the first claim follows. If $n \in N$, then $\mathrm{d}(n)=0$ and therefore it follows that $\sigma_{n \triangleright y}=$ $n \sigma_{y} c\left(n \sigma_{y}\right)^{-1}=\sigma\left(n \sigma_{y}\right) c f$. (3.4).

We can now proceed to prove Theorem 1.2.

Proof of Theorem 1.2 We need to define an inverse to the map (3.10). Fix $a \in A, g \in$ $\operatorname{Hom}\left(N_{0}, A\right)$ and set $f: G \rightarrow M$ as

$$
f(u)(y):=\mathrm{d}(u) a+g\left(c\left(u \sigma_{y} x_{0}^{-\mathrm{d}(u)}\right)\right),
$$

for each $u \in G$. We show that $f \in Z^{1}(G, M)$ and $f \mapsto(a, g)$ via (3.10).

On the one hand, as $\sigma_{x_{0}}=\sigma_{0}=1$,

$$
f\left(x_{0}\right)\left(x_{0}\right)=a+g\left(c\left(x_{0} x_{0}^{-1}\right)\right)=a .
$$

On the other, if $n_{0} \in N_{0}$, then $\mathrm{d}\left(n_{0}\right)=0$ and thus

$$
f_{0}\left(n_{0}\right)=f\left(n_{0}\right)\left(x_{0}\right)=g\left(c\left(n_{0}\right)\right)=g\left(n_{0}\right) .
$$

Now we check the cocycle condition. First,

$$
f\left(u u^{\prime}\right)(y)=\mathrm{d}\left(u u^{\prime}\right) a+g\left(c\left(u u^{\prime} \sigma_{y} x_{0}^{-\mathrm{d}\left(u u^{\prime}\right)}\right)\right) .
$$

Second,

$$
\begin{aligned}
& \left(f(u) \cdot u^{\prime}\right)(y)+f\left(u^{\prime}\right)(y)=f(u)\left(u^{\prime} \triangleright y\right)+f\left(u^{\prime}\right)(y) \\
& \quad=\mathrm{d}(u) a+g\left(c\left(u \sigma_{u^{\prime} \triangleright y} x_{0}^{-\mathrm{d}(u)}\right)\right)+\mathrm{d}\left(u^{\prime}\right) a+g\left(c\left(u^{\prime} \sigma_{y} x_{0}^{-\mathrm{d}\left(u^{\prime}\right)}\right)\right) \\
& \quad=f\left(u u^{\prime}\right)(y),
\end{aligned}
$$

since $A$ is abelian, $\mathrm{d}$ and $g$ are a group homomorphisms and

$$
c\left(u \sigma_{u^{\prime} \triangleright y} x_{0}^{-\mathrm{d}(u)}\right)=c\left(u u^{\prime} \sigma_{y} x_{0}^{-\mathrm{d}\left(u u^{\prime}\right)}\right) c\left(u^{\prime} \sigma_{y} x_{0}^{-\mathrm{d}\left(u^{\prime}\right)}\right)^{-1}
$$

by Lemma 4.3. Hence $f \in Z^{1}(G, M)$. 


\section{Applications}

Our method for computing the 2nd cohomology group of an indecomposable quandle $X$ involves the group $N_{0}$, see Sect. 1.3. In several important cases, this group can be obtained applying the following lemma.

Lemma 5.1 Let $X$ be a finite indecomposable quandle and $x_{0} \in X$. Assume that the canonical map $X \rightarrow G_{X}$ is injective. Then

$$
N_{0} \simeq\left[F_{X}, F_{X}\right] \cap C_{F_{X}}\left(\psi\left(x_{0}\right)\right),
$$

where $\psi: X \rightarrow G_{X} \rightarrow F_{X}$ is the composition of the canonical maps and $C_{F_{X}}\left(\psi\left(x_{0}\right)\right)$ is the centralizer of $\psi\left(x_{0}\right)$ in $F_{X}$.

Proof Since $X \rightarrow G_{X}$ is injective and $X$ is indecomposable, $X$ can be identified with the conjugacy class of $x_{0}$ in $G_{X}$. By [15, Lemma 1.8], $X$ can also be identified with the conjugacy class of $\psi\left(x_{0}\right)$ in $F_{X}$. From Lemma 2.1 one obtains that $N_{X}=\left[G_{X}, G_{X}\right] \simeq\left[F_{X}, F_{X}\right]$ and thus the claim follows.

Remark 5.2 If $X$ is a conjugation quandle, then the canonical map $X \rightarrow G_{X}$ is injective. Thus Lemma 5.1 gives a nice description of $N_{0}$ in the case of finite indecomposable conjugation quandles.

Example 5.3 The claim of Lemma 5.1 does not hold for arbitrary quandles. Let $X$ be the quandle $\left\{x_{1}, x_{2}, x_{3}, x_{4}\right\}$ with the structure given by

$$
\varphi_{x_{1}}=\left(x_{2} x_{3} x_{4}\right), \quad \varphi_{x_{2}}=\left(x_{1} x_{4} x_{3}\right), \quad \varphi_{x_{3}}=\left(x_{1} x_{2} x_{4}\right), \quad \varphi_{x_{4}}=\left(x_{1} x_{3} x_{2}\right) .
$$

This quandle is isomorphic to the conjugacy class of 3-cycles in $\mathbb{A}_{4}$. Let $f: X \times X \rightarrow \mathbb{C}^{\times}$ be the map given by

$$
f(x, y)= \begin{cases}1 & \text { if } x=x_{1} \text { or } y=x_{1} \text { or } x=y, \\ -1 & \text { otherwise. }\end{cases}
$$

Then $f$ is a 2-cocycle of $X$ with values in $\{-1,1\} \simeq \mathbb{Z}_{2}$, see [2, Example 2.2]. Let $Y=$ $X \times\{-1,1\}$ be the quandle given by

$$
(x, i) \triangleright(y, j)=(x \triangleright y, j f(x, y)), \quad x, y \in X, i, j \in\{-1,1\} .
$$

Then the canonical map $Y \rightarrow G_{Y}$ is not injective. Indeed,

$$
\left(x_{2}, 1\right)\left(x_{3},-1\right)=\left(x_{1}, 1\right)\left(x_{2}, 1\right)=\left(x_{3}, 1\right)\left(x_{1}, 1\right)=\left(x_{2}, 1\right)\left(x_{3}, 1\right)
$$

implies that $\left(x_{3},-1\right)=\left(x_{3}, 1\right)$ in $G_{Y}$.

Fix $y_{0} \in Y$. A straighforward calculation shows that $F_{Y} \simeq \mathbf{S L}(2,3)$ and $\left[F_{Y}, F_{Y}\right] \cap$ $C_{F_{Y}}\left(\psi\left(y_{0}\right)\right) \simeq \mathbb{Z}_{2}$. However, since $\left[F_{Y}, F_{Y}\right]$ and $Y$ both have eight elements, $N_{0}$ is the trivial group.

\subsection{Transpositions in $\mathbb{S}_{n}$}

Let $X=(12)^{\mathbb{S}_{n}}$ be the quandle of transpositions in the symmetric group $\mathbb{S}_{n}$. For $n \geq 4 \mathrm{a}$ non-constant 2-cocycle $\chi \in H^{2}\left(X, \mathbb{C}^{\times}\right)$was constructed in [21]. This cocycle is given by

$$
\chi(\sigma, \tau)= \begin{cases}1 & \text { if } \sigma(i)<\sigma(j), \\ -1 & \text { otherwise, }\end{cases}
$$

where $\tau=(i j), 1 \leq i<j \leq n$. 
Lemma 5.4 Let $X=(12)^{\mathbb{S}_{n}}, n \geq 4$, and fix $x_{0}=(12) \in X$.

(1) $F_{X} \simeq \mathbb{S}_{n}$. Hence $N_{X} \simeq \mathbb{A}_{n}$.

(2) $N_{0} \simeq \mathbb{Z}_{2} \ltimes \mathbb{A}_{n-2}$. In particular, $N_{0} /\left[N_{0}, N_{0}\right] \simeq \mathbb{Z}_{2}$.

Proof Recall that $\mathbb{S}_{n}=\left\langle\sigma_{1}, \ldots, \sigma_{n-1}\right\rangle$ with relations

$$
\begin{aligned}
& \sigma_{i} \sigma_{i+1} \sigma_{i}=\sigma_{i+1} \sigma_{i} \sigma_{i+1}, \quad 1 \leq i<n-1, \\
& \sigma_{k} \sigma_{j}=\sigma_{j} \sigma_{k}, \quad 1 \leq j, k<n,|j-k|>1, \\
& \sigma_{i}^{2}=1, \quad 1 \leq i<n .
\end{aligned}
$$

Set $\iota: X \hookrightarrow \mathbb{S}_{n}$ the canonical inclusion let $\varphi:\langle X\rangle \rightarrow \mathbb{S}_{n}$ the unique group homomorphism with $\varphi_{\mid X}=\iota$. This is in fact an epimorphism. Observe that $\varphi\left(x^{2}\right)=\iota(x)^{2}=1$ and

$$
\varphi(x y)=\iota(x) \iota(y)=\iota(x) \iota(y) \iota(x)^{-1} \iota(x)=\iota(x \triangleright y) \iota(x)=\varphi((x \triangleright y) x) .
$$

Thus, $\varphi$ factors through $\phi: F_{X} \rightarrow \mathbb{S}_{n}$. Now, set $S$ be the free group on $s_{1}, \ldots, s_{n-1}$ and let $\psi^{\prime}: S \rightarrow F_{X}$ be the group epihomomorphism given by $s_{i} \mapsto(i i+1)$. Now $\psi^{\prime}$ factors through $\psi: \mathbb{S}_{n} \rightarrow F_{X}$ and it is clear that $\phi$ and $\psi$ are inverses to each other.

Let us prove the second claim. By the first part, we identify $N$ with $\mathbb{A}_{n}$. Consider $\mathbb{A}_{n-2} \leq$ $\mathbb{A}_{n}$ as those permutations fixing 1 and 2 and set $t=(12)(34)$. Then $t \sigma t^{-1} \in \mathbb{A}_{n-2}$ for all $\sigma \in \mathbb{A}_{n-2}$. Clearly $\langle t\rangle \ltimes \mathbb{A}_{n-2} \leq N_{0}$.

Since $\mathbb{A}_{n}$ is generated by $\{(34 \ell) \mid 1 \leq \ell \leq n, \ell \neq 3,4\}$, the group $N$ is generated by the subgroups $\mathbb{A}_{n-2}$ and $\mathbb{A}_{4} \simeq\langle(134),(234)\rangle$. Notice that $\langle(134),(234)\rangle \cap N_{0} \simeq\langle t\rangle$. We have $\left|\langle t\rangle \ltimes \mathbb{A}_{n-2}\right|=(n-2)$ ! and

$$
\left\{\sigma(12) \sigma^{-1}: \sigma \in \mathbb{A}_{n}\right\}=(12)^{\mathbb{S}_{n}} .
$$

Thus $\left|N_{0}\right|=|N| /\left|(12)^{\mathbb{S}_{n}}\right|=(n-2)$ ! and hence $N_{0}=\langle t\rangle \ltimes \mathbb{A}_{n-2}$.

Finally, since the commutator subgroup of some group $A \ltimes B$ is the group generated by $[A, A] \cup[A, B] \cup[B, B]$ and $N_{0}=\langle t\rangle \ltimes \mathbb{A}_{n-2}$, it follows that $\left[N_{0}, N_{0}\right] \simeq \mathbb{A}_{n-2}$ and hence $N_{0} /\left[N_{0}, N_{0}\right] \simeq \mathbb{Z}_{2}$

Theorem 5.5 Let $n \geq 4$ and $X=(12)^{\mathbb{S}_{n}}$ be the conjugacy class of transpositions. Then $H^{2}\left(X, \mathbb{C}^{\times}\right) \simeq \mathbb{C}^{\times} \times\langle\chi\rangle$.

Proof Set $x_{0}=(12) \in \mathbb{S}_{n}$. Since $N_{0} \simeq \mathbb{Z}_{2} \ltimes \mathbb{A}_{n-2}$ and $N_{0} /\left[N_{0}, N_{0}\right] \simeq \mathbb{Z}_{2}$ by Lemma 5.4, it follows that $\operatorname{Hom}\left(N_{0}, \mathbb{C}^{\times}\right) \simeq \mathbb{Z}_{2}$. Applying the isomorphism (1.3) of Theorem 1.1 to the 2-cocycle $\chi$ given in (5.1),

$$
\chi \mapsto\left(-1,\left(f_{\chi}\right)_{0}\right),
$$

where $\left(f_{\chi}\right)_{0}: N_{0} \rightarrow \mathbb{C}^{\times}, n_{0} \mapsto f_{\chi}\left(n_{0}\right)\left(x_{0}\right), n_{0} \in N_{0}$. Now the claim follows since $\left(f_{\chi}\right)_{0}$ generates $\operatorname{Hom}\left(N_{0}, \mathbb{C}^{\times}\right)$. Indeed, $f_{\chi} \neq 1$ since

$$
\begin{aligned}
\left(f_{\chi}\right)_{0}((12)(34)) & =f_{\chi}((12)(34))(12) \stackrel{(2.3)}{=} \chi((12),(34) \triangleright(12)) \chi((34),(12)) \\
& =\chi((12),(12)) \chi((34),(12))=-1 .
\end{aligned}
$$

This completes the proof. 


\subsection{Eisermann formula}

We give a new proof of a formula of Eisermann as a consequence of our results.

Theorem 5.6 [8, Theorem 1.12] Let $X$ be a finite indecomposable quandle and $x_{0} \in X$. Then

$$
H_{2}^{Q}(X, \mathbb{Z}) \simeq\left(\left[G_{X}, G_{X}\right] \cap C_{G_{X}}\left(x_{0}\right)\right)_{\mathrm{ab}} \simeq\left(N_{0}\right)_{\mathrm{ab}},
$$

where $N_{0}$ is the stabilizer of a given $x_{0} \in X$ of the action of $\left[G_{X}, G_{X}\right]$ on $X$.

Proof The claim follows by "chasing" the chain of equivalences

$$
A \times \operatorname{Hom}\left(N_{0}, A\right) \simeq H^{2}(X, A) \simeq \operatorname{Hom}\left(H_{2}(X, \mathbb{Z}), A\right)
$$

given by the application of Theorem 1.2 and Lemma 2.5. More explicitly, if $(a, g) \in A \times$ $\operatorname{Hom}\left(N_{0}, A\right)$, then it defines $q \in H^{2}(X, A)$ via (1.4), which in turn defines a morphism $H_{2}(X, \mathbb{Z}) \rightarrow A$ by Lemma 2.5:

$$
[x, y] \mapsto q_{x, y}=a+g\left(c\left(x \sigma_{y} x_{0}^{-1}\right)\right) \in A,
$$

cf. Theorem 1.2. Now, $H_{2}(X, \mathbb{Z}) \simeq H_{2}^{Q}(X, \mathbb{Z}) \times \mathbb{Z}$ by Lemma 2.6 and so this assignment becomes a map in $\operatorname{Hom}\left(H_{2}^{Q}(X, \mathbb{Z}) \times \mathbb{Z}, A\right)$ :

$$
([x, y], \ell) \longmapsto \ell a+g\left(c\left(x \sigma_{y} x_{0}^{-1}\right)\right) .
$$

Thus we see that the restriction of this map to $H_{2}^{Q}(X, \mathbb{Z}) \times\{0\}$ gives an equivalence $\operatorname{Hom}\left(H_{2}^{Q}(X, \mathbb{Z}), A\right) \simeq \operatorname{Hom}\left(N_{0}, A\right) \simeq\left(N_{0}\right)_{\mathrm{ab}}$ for any abelian group $A$. Hence we derive Eisermann's formula $H_{2}^{Q}(X, \mathbb{Z}) \simeq\left(N_{0}\right)_{\mathrm{ab}}$.

If we combine this fact with Lemma 2.6, we obtain the following.

Corollary 5.7 Let $X$ be a finite indecomposable quandle, $x_{0} \in X$. Then $H_{2}(X, \mathbb{Z}) \simeq$ $\left(N_{0}\right)_{\mathrm{ab}} \times \mathbb{Z}$.

\subsection{Affine quandles}

Let $L$ be an abelian group and $\gamma \in \operatorname{Aut}(L)$. The affine (or Alexander) quandle $\operatorname{Aff}(L, \gamma)$ is the set $L$ together with the action

$$
x \triangleright y=\gamma(y)+x-\gamma(x), \quad x, y \in L .
$$

In [7] Clauwens described the enveloping group of an affine quandle; we review his construction next. Set

$$
\begin{aligned}
\tau_{\gamma}: L \otimes_{\mathbb{Z}} L \rightarrow L \otimes_{\mathbb{Z}} L, \quad(x, y) \mapsto(x, y)-(y, \gamma(x)), \\
S(L, \gamma):=\operatorname{coker} \tau_{\gamma}=L \otimes_{\mathbb{Z}} L /\langle(x, y)-(y, \gamma(x))\rangle .
\end{aligned}
$$

We write $[x, y] \in S(L, \gamma)$ for the class of an element $x \otimes y \in L \otimes_{\mathbb{Z}} L$. Set $X=\operatorname{Aff}(L, \gamma)$; then $G_{X}$ is the set $L \rtimes \mathbb{Z} \times S(L, \gamma)$ with multiplication

$$
(x, m,[p, q])(y, n,[r, s])=\left(x+\gamma^{m}(y), m+n,\left[p+r+x, q+s+\gamma^{m}(y)\right]\right),
$$

for $m, n \in \mathbb{Z}, x, y \in L,[p, q],[r, s] \in S(L, \gamma)$. 
The rack $X$ identifies with the subset $L \rtimes\{1\} \times 0$ with the rack action given by conjugation:

$$
\begin{aligned}
(x, 1,0)(y, 1,0) & =(x+\gamma(y), 2,[x, \gamma(y)])=(x+\gamma(y), 2,[x \triangleright y, \gamma(x)]) \\
& =(x+\gamma(y)-\gamma(x)+\gamma(x), 2,[x \triangleright y, \gamma(x)]) \\
& =(x \triangleright y, 1,0)(x, 1,0)
\end{aligned}
$$

since $[x \triangleright y, \gamma(x)]=[\gamma(y), \gamma(x)]+[x, \gamma(x)]-[\gamma(x), \gamma(x)]=[x, \gamma(y)]$, as $[x, \gamma(x)]=$ $[\gamma(x), \gamma(x)]$. We fix $x_{0}=(0,1,0)$; then

$$
N_{X}=L \times\{0\} \times S(L, \gamma), \quad N_{0}=\{0\} \times\{0\} \times S(L, \gamma) .
$$

Let $\left\{x_{0}, x_{1}, \ldots, x_{n}\right\}$ be an enumeration of the elements of $L$. In particular,

$$
N_{X}=\bigsqcup_{i \in\{0, \ldots, n\}} \sigma_{i} N_{0} \simeq L \times \text { coker } \tau_{\gamma}, \quad \sigma_{i}=\left(x_{i}, 0,0\right),
$$

is a good decomposition of $N$ into $N_{0}$-cosets, $c f$. Proposition 4.1. Indeed,

(1) $\sigma_{0}=(0,0,0)$ coincides with the unit element in $G_{X}$;

(2) fix $j \in\{0, \ldots, n\}$ and let $k \in\{0, \ldots, n\}$ be such that $x_{k}=\gamma\left(x_{j}\right)$. Then $x_{0} \triangleright \sigma_{j}=$ $(0,1,0)\left(x_{j}, 0,0\right)(0,-1,0)=\left(\gamma\left(x_{j}\right), 0,0\right)=\sigma_{k} ;$ and

(3) if $i \in\{0, \ldots, n\}$ and $x_{j}=(1-\gamma)^{-1}\left(x_{i}\right)$, then $\sigma_{j} \triangleright x_{0}=x_{i}$.

Recall from (4.1) the definition of the elements $\sigma_{y}, y \in X$, and from (3.4) the map $c: N_{X} \rightarrow$ $N_{0}$. We see from Item (3) above that in this case

$$
\sigma_{y}=\left((1-\gamma)^{-1}(y), 0,0\right), \quad y \in X .
$$

As a direct consequence of Theorem 1.2, we obtain the following.

Proposition 5.8 Let $L$ be an abelian group, $\gamma \in \operatorname{Aut}(L)$ and $X=\operatorname{Aff}(L, \gamma)$ be the corresponding affine quandle and set $\Gamma=S(L, \gamma)$ as in (5.2). Fix $x_{0}=0 \in X$ and let $A$ be an abelian group with trivial $G_{X}$-action. Consider a decomposition of $N_{X}$ into $N_{0}$-cosets as in (5.4). For each $a \in A$ and $g \in \operatorname{Hom}(\Gamma, A)$, the map $q: X \times X \rightarrow A$ given by

$$
q_{x, y}=a+\sum_{0<j<\operatorname{ord}(\gamma)} g\left(\left[x, \gamma^{j}(y)\right]\right)
$$

is a 2-cocycle of $X$ and any $q \in H^{2}(X, A)$ arises in this way.

Proof By Theorem 1.2 and Corollary 1.3, any 2-cocycle is of the form

$$
q_{x, y}=a+g\left(c\left(x \sigma_{y} x_{0}^{-1}\right)\right) .
$$

for some $a \in A$ and $g \in \operatorname{Hom}(\Gamma, A)$. Using the identifications above, we have

$$
\begin{aligned}
x \sigma_{y} x_{0}^{-1} & =(x, 1,0)\left((1-\gamma)^{-1}(y), 0,0\right)(0,-1,0) \\
& =\left(x+\gamma(1-\gamma)^{-1}(y), 0,\left[x, \gamma(1-\gamma)^{-1}(y)\right]\right) \\
& =\sigma_{k}\left(0,0,\left[x, \gamma(1-\gamma)^{-1}(y)\right]\right) \in \sigma_{k} N_{0}
\end{aligned}
$$

for $k \in\{0, \ldots, n\}$ such that $x+\gamma(1-\gamma)^{-1}(y)=x_{k}$. Hence

$$
\gamma(1-\gamma)^{-1}(y)=(1-\gamma)^{-1}(y)-y=\sum_{0<j<\operatorname{ord}(\gamma)} \gamma^{j}(y)
$$

and the result follows. 
If $L=\mathbb{F}_{q}$ is the finite field of $q$ elements and $\gamma$ is the multiplication by some $1 \neq \omega \in \mathbb{F}_{q}^{\times}$, we write $\operatorname{Aff}(q, \omega)=\operatorname{Aff}(L, \gamma)$.

Lemma 5.9 Let $p$ be a prime number and $1 \neq \omega \in \mathbb{F}_{p}^{\times}$, set $X=\operatorname{Aff}(p, \omega)$. Then $G_{X} \simeq$ $L \rtimes \mathbb{Z}, N_{X} \simeq L$ and $N_{0}$ is trivial.

Proof Indeed, $S(L, \gamma)$ is a quotient of $\mathbb{Z}_{p} \simeq \mathbb{Z}_{p} \otimes_{\mathbb{Z}} \mathbb{Z}_{p}$ and we have that $0 \neq(1-\omega) \otimes 1 \in$ $\operatorname{Im}\left(\tau_{\gamma}\right)$, hence $S(L, \gamma)=0$ and the lemma follows.

We recover the following result from [13, Lemma 5.1].

Proposition 5.10 $H^{2}\left(\operatorname{Aff}(p, \omega), \mathbb{C}^{\times}\right) \simeq \mathbb{C}^{\times}$.

Proof It follows from Theorem 1.1, using Lemma 5.9.

\subsection{Indecomposable quandles of size $p^{2}$}

Let $p$ be a prime number and let $X$ be an indecomposable quandle of size $p^{2}$. By [13], $X$ is one of the following affine quandles $(L, \gamma)$ in the following list:

$$
\begin{aligned}
& L=\mathbb{Z}_{p} \oplus \mathbb{Z}_{p}, \quad \gamma_{\alpha, \beta}(x, y)=(\alpha x, \beta y), \quad \alpha, \beta \in \mathbb{Z}_{p}^{*} \backslash\{1\} ; \\
& L=\mathbb{Z}_{p} \oplus \mathbb{Z}_{p}, \quad \gamma_{\alpha}(x, y)=(\alpha x, \alpha y+x), \quad \alpha \in \mathbb{Z}_{p}^{*} \backslash\{1\} ; \\
& L=\mathbb{F}_{p^{2}}, \quad \gamma_{\alpha}(x)=\alpha x, \quad \alpha \in \mathbb{F}_{p^{2} \backslash \mathbb{F}_{p} ;} ; \\
& L=\mathbb{Z}_{p^{2}}, \quad \gamma_{\alpha}(x)=\alpha x, \quad \alpha \neq 0,1(p) .
\end{aligned}
$$

We identify $\mathbb{F}_{p^{2}} \simeq \mathbb{F}_{p} \oplus \mathbb{F}_{p}$ as abelian groups for notational reasons. For $\alpha=\left(\alpha_{0}, \alpha_{1}\right) \in \mathbb{F}_{p}^{2}$ we set

$$
d_{\alpha}:=\left(1-\alpha_{0}+\alpha_{1}\right)\left(1-\alpha_{0}-\alpha_{1}\right)\left(1-\alpha_{0}^{2}+\alpha_{1}^{2}\right) .
$$

Assume $\alpha \in \mathbb{F}_{p^{2}} \backslash \mathbb{F}_{p}$, so $\alpha_{1} \neq 0$. If $d_{\alpha}=0$, then $\alpha_{0} \neq 1$ and we set

$$
t_{\alpha}:=\left(\alpha_{0}-\alpha_{0}^{2}+\alpha_{1}^{2}\right)\left(1-\alpha_{0}\right)^{-1}, \quad s_{\alpha}:=\left(1-\alpha_{0}\right) \alpha_{1}^{-1} .
$$

Proposition 5.11 The 2nd homology groups of the indecomposable quandles of order $p^{2}$ are as follows:

$$
\begin{aligned}
H_{2}\left(\left(\mathbb{Z}_{p} \oplus \mathbb{Z}_{p}, \gamma_{\alpha, \beta}\right), \mathbb{Z}\right) & \simeq \begin{cases}\mathbb{Z} \times \mathbb{Z}_{p}, & \text { if } \alpha \beta=1, \\
\mathbb{Z}, & \text { if } \alpha \beta \neq 1 .\end{cases} \\
H_{2}\left(\left(\mathbb{Z}_{p} \oplus \mathbb{Z}_{p}, \gamma_{\alpha}\right), \mathbb{Z}\right) & \simeq \begin{cases}\mathbb{Z} \times \mathbb{Z}_{p}, & \text { if } \alpha^{2}=1, \\
\mathbb{Z}, & \text { if } \alpha^{2} \neq 1 .\end{cases} \\
H_{2}\left(\left(\mathbb{F}_{p^{2}}, \gamma_{\alpha}\right), \mathbb{Z}\right) & \simeq \begin{cases}\mathbb{Z} \times \mathbb{Z}_{p}, & \text { if } d_{\alpha}=0, \\
\mathbb{Z}, & \text { if } d_{\alpha} \neq 0 .\end{cases} \\
H_{2}\left(\left(\mathbb{Z}_{p^{2}}, \gamma_{\alpha}\right), \mathbb{Z}\right) & \simeq \mathbb{Z} .
\end{aligned}
$$

Proof By Corollary (5.7), if $X=(L, \gamma)$ and $\tau_{\gamma}: L \otimes_{\mathbb{Z}} L \rightarrow L \otimes_{\mathbb{Z}} L$ as in (5.2), then

$$
H_{2}(X, \mathbb{Z})=\left(N_{0}\right)_{\mathrm{ab}} \times \mathbb{Z}=\text { coker } \tau_{\gamma} \times \mathbb{Z}
$$


We compute coker $\tau_{\gamma}$ case by case. We will use the identifications

$$
\begin{aligned}
&\left(\mathbb{Z}_{p} \oplus \mathbb{Z}_{p}\right) \otimes_{\mathbb{Z}}\left(\mathbb{Z}_{p} \oplus \mathbb{Z}_{p}\right) \simeq \mathbb{Z}_{p}^{4}, \quad(a, b) \otimes(c, d) \mapsto(a c, a d, b c, b d) \\
& \mathbb{F}_{p^{2}} \otimes_{\mathbb{Z}} \mathbb{F}_{p^{2}} \simeq \mathbb{F}_{p}^{2} \otimes_{\mathbb{F}_{p}} \mathbb{F}_{p}^{2} \simeq \mathbb{F}_{p}^{4}, \quad(a, b) \otimes(c, d) \mapsto(a c, a d, b c, b d) \\
& \mathbb{Z}_{p^{2}} \otimes_{\mathbb{Z}} \mathbb{Z}_{p^{2}} \simeq \mathbb{Z}_{p^{2}}, \quad a \otimes b \mapsto a b .
\end{aligned}
$$

Case (5.6): We have that $\tau_{\alpha, \beta}:=\tau_{\gamma_{\alpha, \beta}}$ is

$$
\tau_{\alpha, \beta}((a, b) \otimes(c, d))=(a, b) \otimes(c, d)-(c, d) \otimes(\alpha a, \beta b) .
$$

With the identifications above this yields

$$
\tau_{\alpha, \beta}: \mathbb{Z}_{p}^{4} \rightarrow \mathbb{Z}_{p}^{4}, \quad(x, y, z, w) \mapsto((1-\alpha) x, y-\beta z, z-\alpha y,(1-\beta) w) .
$$

Next, we compute the image $I_{\alpha, \beta}$ of this map: For $(a, b, c, d) \in \mathbb{Z}_{p}^{4}$ to be in this subgroup, we need $x=a(1-\alpha)^{-1}, w=d(1-\beta)^{-1}$ (recall $\alpha, \beta \neq 1$ ) and $y, z$ to be a solution of $y-\beta z=b,-\alpha y+z=c$. This system has always a solution if $\alpha \beta \neq 1$. If $\alpha \beta=1$, then

$$
\begin{aligned}
I_{\alpha, \beta} & =\left\{(a, b,-\alpha b, d) \mid a, b, d \in \mathbb{Z}_{p}\right\} \simeq \mathbb{Z}_{p}^{3}, \text { hence } \\
\text { coker } \tau_{\alpha} & = \begin{cases}0, & \text { if } \alpha \beta \neq 1, \\
\mathbb{Z}_{p}, & \text { if } \alpha \beta=1 .\end{cases}
\end{aligned}
$$

In case (5.7), we have $\tau_{\alpha}:=\tau_{\gamma_{\alpha}}: \mathbb{Z}_{p}^{4} \rightarrow \mathbb{Z}_{p}^{4}$ is given by

$$
(x, y, z, w) \mapsto((1-\alpha) x, y-\alpha z+x, z-\alpha y,(1-\alpha) w-y) .
$$

For $(a, b, c, d)$ to be in the image $I_{\alpha}$ of $\tau_{\alpha}$, we need $x=a(1-\alpha)^{-1}($ recall $\alpha \neq 1)$ and $(y, z, w)$ to be a solution of

$$
y-\alpha z=b-a(1-\alpha)^{-1}, \quad-\alpha y+z=c, \quad-y+(1-\alpha) w=d .
$$

This system has always a solution if $\alpha^{2} \neq 1$. If $\alpha^{2}=1$, then

$$
\begin{aligned}
I_{\alpha} & =\left\{(a, b, \alpha b-\alpha(1-\alpha) a, d) \mid a, b, d \in \mathbb{Z}_{p}\right\} \simeq \mathbb{Z}_{p}^{3}, \text { hence } \\
\text { coker } \tau_{\alpha} & = \begin{cases}0, & \text { if } \alpha^{2} \neq 1, \\
\mathbb{Z}_{p}, & \text { if } \alpha^{2}=1 .\end{cases}
\end{aligned}
$$

In case (5.8), if $\alpha=\left(\alpha_{0}, \alpha_{1}\right) \in \mathbb{F}_{p}^{2} \backslash \mathbb{F}_{p}$ (hence $\alpha_{1} \neq 0$ ), then the map $\tau_{\alpha} \in \operatorname{End}\left(\mathbb{F}_{p}^{4}\right)$ is represented by the matrix

$$
\left[\tau_{\alpha}\right]=\left(\begin{array}{cccc}
1-\alpha_{0} & 0 & -\alpha_{1} & 0 \\
-\alpha_{1} & 1 & -\alpha_{0} & 0 \\
0 & -\alpha_{0} & 1 & -\alpha_{1} \\
0 & -\alpha_{1} & 0 & 1-\alpha_{0}
\end{array}\right),
$$

with $\operatorname{det}\left[\tau_{\alpha}\right]=d_{\alpha}$, see (5.10). Let $I_{\alpha}$ denote the image of this map. Now, the rank of this matrix is $\geq 3$, as $\operatorname{det}\left(\begin{array}{ccc}0 & -\alpha_{1} & 0 \\ 1 & -\alpha_{0} & 0 \\ -\alpha_{0} & 1 & -\alpha_{1}\end{array}\right)=-\alpha_{1}^{2} \neq 0$. Hence,

$$
\text { coker } \tau_{\alpha}= \begin{cases}0, & \text { if } \operatorname{det}\left[\tau_{\alpha}\right] \neq 0 \\ \mathbb{Z}_{p}, & \text { if } \operatorname{det}\left[\tau_{\alpha}\right]=0\end{cases}
$$


If $\operatorname{det}\left[\tau_{\alpha}\right]=0$, i.e. $\mathrm{d}_{\alpha}=0$, then we set $t_{\alpha}, s_{\alpha} \in \mathbb{F}_{p}$ as in (5.11) and thus

$$
I_{\alpha}=\left\{\left(a, b, c,-t_{\alpha}(a+b)-s_{\alpha} c\right) \mid a, b, c \in \mathbb{Z}_{p}\right\} \simeq \mathbb{Z}_{p}^{3} .
$$

In case (5.9), $\tau_{\alpha}: \mathbb{Z}_{p^{2}} \rightarrow \mathbb{Z}_{p^{2}}$ is $x \mapsto(1-\alpha) x$; hence coker $\tau_{\alpha}=0$.

\subsection{Explicit cocycles}

Next we apply Proposition 5.8 to compute all non-constant 2-cocycles for the affine quandles $X$ described in (5.6)-(5.9). More precisely, we focus on those affine quandles in that list admitting a non-constant 2-cocyle, as stated in Proposition 5.11:

$$
\begin{aligned}
& L=\mathbb{Z}_{p} \oplus \mathbb{Z}_{p}, \quad \gamma_{\alpha}(x, y)=\left(\alpha x, \alpha^{-1} y\right), \quad \alpha \in \mathbb{Z}_{p}^{*} \backslash\{1\} ; \\
& L=\mathbb{Z}_{p} \oplus \mathbb{Z}_{p}, \quad \gamma(x, y)=(-x, x-y) ; \\
& L=\mathbb{F}_{p^{2}}, \quad \gamma_{\alpha}(x)=\alpha x, \quad \alpha \in \mathbb{F}_{p^{2} \backslash \mathbb{F}_{p}, d_{\alpha}=0 .}
\end{aligned}
$$

Recall our identification $\mathbb{F}_{p^{2}} \simeq \mathbb{F}_{p}^{2}, x \mapsto\left(x_{0}, x_{1}\right)$, and $t_{\alpha}, s_{\alpha} \in \mathbb{Z}_{p}$ from (5.11). For $x, y \in L$ and $j \in \mathbb{N}$ we set, for $X$ is as in (5.13),

$$
\zeta_{j}(x, y)=\alpha^{j} x_{2} y_{1}+\alpha^{1-j} x_{1} y_{2}
$$

for $X$ is as in (5.14),

$$
\zeta_{j}(x, y)=\left(j+2(-1)^{j}\right) x_{1} y_{1}+(-1)^{j}\left(x_{1} y_{2}-x_{2} y_{1}\right) ;
$$

and, for $X$ is as in (5.15),

$$
\zeta_{j}(x, y)=x_{1}\left(\alpha^{j} y\right)_{1}+t_{\alpha}\left(x_{0}\left(\alpha^{j} y\right)_{0}+x_{0}\left(\alpha^{j} y\right)_{1}\right)+s_{\alpha} x_{1}\left(\alpha^{j} y\right)_{0} .
$$

Next, we define the map $\langle\rangle:, L \times L \rightarrow \mathbb{Z}$ as

$$
\langle x, y\rangle=\sum_{0<j<\operatorname{ord}(\gamma)} \zeta_{j}(x, y), \quad x, y \in L .
$$

Notice that $\operatorname{ord}(\gamma)=p-1,2 p$ (or 2 if $p=2$ ) or $p^{2}-1$ according to whether $X$ is as in (5.13), (5.14) or (5.15), respectively.

Proposition 5.12 Let $X=(L, \gamma)$ be an indecomposable affine rack of order $p^{2}$. If $q \in$ $H^{2}\left(X, \mathbb{k}^{*}\right)$ is non-constant, then $X$ belongs to the list (5.13)-(5.15) and there are $0<\ell<p$ and $\lambda \in \mathbb{R}^{*}$ such that

$$
q_{x, y}=\lambda \exp \left(\frac{2 \pi i \ell}{p}\langle x, y\rangle\right), \quad x, y \in X .
$$

Proof Fix $x_{0}=0 \in L$ and a good decomposition $N \simeq L \times$ coker $\tau_{\gamma}$ of $N_{X}$ into $N_{0}$-cosets, see (5.4). In this case, $N_{0}=x_{0} \times$ coker $\tau_{\gamma} \simeq \mathbb{Z}_{p}$, by Proposition 5.11. More precisely, if we denote by $\varphi: N_{0} \rightarrow \mathbb{Z}_{p}$ this isomorphism, then it follows from the proof of Proposition 5.11 that, for $t_{\alpha}, s_{\alpha}$ as in (5.11):

$$
\varphi([(a, b),(c, d)])= \begin{cases}b c+\alpha a d \in \mathbb{Z}_{p}, & X \text { as (5.13); } \\ b c+a d-2 a c \in \mathbb{Z}_{p}, & X \text { as (5.14); } \\ b d+t_{\alpha}(a c+a d)+s_{\alpha} b c \in \mathbb{Z}_{p}, & X \text { as (5.15) }\end{cases}
$$


On the other hand, if $g \in \operatorname{Hom}\left(N_{0}, \mathbb{k}^{*}\right)$, then there is $0 \leq \ell<p$ such that $g$ is the morphism $g_{\ell}$ given by $1 \mapsto \exp \left(\frac{2 \pi \mathrm{i} \ell}{p}\right)$. By Proposition 5.8, any $q \in H^{2}\left(X, \mathbb{k}^{*}\right)$ is thus of the form

$$
q_{x, y}=\lambda \prod_{0<j<\operatorname{ord}(\gamma)} \exp \left(\frac{2 \pi \mathrm{i} \ell}{p} \varphi\left(\left[x, \gamma^{j}(y)\right]\right)\right), \quad x, y \in X,
$$

for some $\lambda \in \mathbb{k}^{*}, \ell \in \mathbb{Z}$. Hence the result follows as $\zeta_{j}(x, y) \in \mathbb{Z}$ is a representative of $\varphi\left(\left[x, \gamma^{j}(y)\right]\right)$, for each $x, y \in X$, via (5.17).

Acknowledgements We thank G. García and M. Kotchetov for interesting discussions. We also thank N. Andruskiewitsch for his constant guidance and support. This work was initiated while the authors were visiting María Ofelia Ronco, at Universidad de Talca, Chile. We are grateful for her warm hospitality. The authors are grateful to the reviewer for useful remarks, interesting suggestions and corrections.

\section{References}

1. Andruskiewitsch, N., Fantino, F., García, G.A., Vendramin, L.: On Nichols algebras associated to simple racks. In: Milies, C.P. (ed.) Groups, Algebras and Applications, volume 537 of Contemporary Mathematics pp. 31-56. American Mathematical Society, Providence, RI (2011)

2. Andruskiewitsch, N., Graña, M.: From racks to pointed Hopf algebras. Adv. Math. 178(2), 177-243 (2003)

3. Brown, K.S.: Cohomology of Groups, volume 87 of Graduate Texts in Mathematics. Springer, New York, Corrected reprint of the 1982 original (1994)

4. Carter, J.S., Jelsovsky, D., Kamada, S., Langford, L., Saito, M.: Quandle cohomology and state-sum invariants of knotted curves and surfaces. Trans. Amer. Math. Soc. 355(10), 3947-3989 (2003)

5. Carter, J.S., Jelsovsky, D., Kamada, S., Saito, M.: Quandle homology groups, their Betti numbers, and virtual knots. J. Pure Appl. Algebra 157(2-3), 135-155 (2001)

6. Clark, W.E., Elhamdadi, M., Saito, M., Yeatman, T.: Quandle colorings of knots and applications. J. Knot Theory Ramif. 23(6), 1450035 (2014)

7. Clauwens, F.J.B.J.: The Adjoint Group of an Alexander Quandle. Preprint: arXiv:1011.1587

8. Eisermann, M.: Quandle coverings and their Galois correspondence. Fund. Math. 225, 103-168 (2014)

9. Etingof, P., Graña, M.: On rack cohomology. J. Pure Appl. Algebra 177(1), 49-59 (2003)

10. Fenn, R., Rourke, C., Sanderson, B.: Trunks and classifying spaces. Appl. Categ. Structures 3(4), 321-356 (1995)

11. Fenn, R., Rourke, C., Sanderson, B.: James bundles. Proc. Lond. Math. Soc. (3) 89(1), 217-240 (2004)

12. Graña, M.: On Nichols algebras of low dimension. In: Andruskiewitsch, N., Ferrer Santos, W.R., Schneider, H-J. (eds.) New Trends in Hopf Algebra Theory (La Falda, 1999), volume 267 of Contemp. Math., pp. 111-134. Amer. Math. Soc., Providence, RI (2000)

13. Graña, M.: Indecomposable racks of order $p^{2}$. Beiträge Algebra Geom. 45(2), 665-676 (2004)

14. Graña, M., Heckenberger, I., Vendramin, L.: Nichols algebras of group type with many quadratic relations. Adv. Math. 227(5), 1956-1989 (2011)

15. Heckenberger, I., Vendramin, L.: Nichols algebras over groups with finite root system of rank two II. J. Group Theory 17(6), 1009-1034 (2014)

16. Hulpke, A., Stanovský, D., Vojtěchovský, P.: Connected quandles and transitive groups. J. Pure Appl. Algebra 220(2), 735-758 (2016)

17. Joyce, D.: A classifying invariant of knots, the knot quandle. J. Pure Appl. Algebra 23(1), 37-65 (1982)

18. Lebed, V.: Homologies of algebraic structures via braidings and quantum shuffles. J. Algebra 391, 152192 (2013)

19. Litherland, R.A., Nelson, S.: The Betti numbers of some finite racks. J. Pure Appl. Algebra 178(2), 187-202 (2003)

20. Matveev, S.V.: Distributive groupoids in knot theory. Mat. Sb. (N.S.), 119(161)(1), 78-88, 160 (1982)

21. Milinski, A., Schneider, H.-J.: Pointed indecomposable Hopf algebras over Coxeter groups. In: Andruskiewitsch, N., Ferrer Santos, W.R., Schneider, H-J. (eds.) New trends in Hopf Algebra Theory (La Falda, 1999), volume 267 of Contemporary Mathematics, pp. 215-236. American Mathematical Society, Providence, RI (2000)

22. Nosaka, T.: Quandle homotopy invariants of knotted surfaces. Math. Z. 274(1-2), 341-365 (2013) 
23. Rotman, J.J.: An introduction to the theory of groups, volume 148 of Graduate Texts in Mathematics. Springer, New York, 4th edition (1995)

24. Stanovský, D.: A guide to self-distributive quasigroups, or Latin quandles. Quasigroups Relat. Syst. 23(1), 91-128 (2015)

25. Tamaru, H.: Two-point homogeneous quandles with prime cardinality. J. Math. Soc. Jpn. 65(4), 11171134 (2013) 